\title{
Geheilt, aber nicht gesund: Das Problem der Langzeitnebenwirkungen
}

\author{
Markus Horneber $^{\mathrm{a}}$ Diana Lüftner ${ }^{\mathrm{b}}$ Roman Pfister ${ }^{\mathrm{c}}$ \\ a Universitätskliniken für Innere Medizin 3 und 5, Schwerpunkte Pneumologie und Onkologie/Hämatologie, Paracelsus Medizinische Privatuniversität, Klini- \\ kum Nürnberg, Nürnberg, Deutschland; b Medizinische Klinik mit Schwerpunkt Hämatologie, Onkologie und Tumorimmunologie, Charité Campus Benjamin \\ Franklin - Universitätsmedizin Berlin, Berlin, Deutschland; ' ${ }^{c}$ Klinik III für Innere Medizin, Universitätsklinikum Köln, Köln, Deutschland.
}

\section{Problemfeld Fertilität}

\section{Referentin: Diana Lüftner, Berlin}

Die meisten jungen Menschen mit Krebs haben heute gute Heilungschancen. Dies erfordert aber meist eine intensive medikamentöse Therapie, oft zusammen mit einer Strahlentherapie oder (etwa beim Mammakarzinom) mit einer Hormontherapie. In der Altersgruppe der 18-39-Jährigen erkranken pro Jahr etwa 15000 Personen an Krebs. Die Ängste der Betroffenen sind vielfältig, dabei stehen Sorgen um die finanzielle Situation und die Familienplanung im Vordergrund, wie eine repräsentative Studie aus Leipzig gezeigt hat [1]. Um die Bewältigung der spezifischen Probleme dieser Altersgruppe, insbesondere in Bezug auf die Therapiefolgen, geht es der Deutschen Stiftung für junge Erwachsene mit Krebs. Eine von der Deutschen Gesellschaft für Hämatologie und Medizinische Onkologie (DGHO) gemeinsam mit der Stiftung durchgeführte Umfrage unter Teilnahme von 31 Frauen und 6 Männern hat gezeigt, dass auf Fragen zum Erhalt der Fruchtbarkeit von ärztlicher Seite oft unzureichend eingegangen wird [2].

Strahlentherapien und zahlreiche Zytostatika schädigen gesundes hochproliferatives Gewebe und können die Fertilität einschränken, indem sie die Eizellen der Frau und die für die Spermienbildung benötigten Stammzellen beim Mann schädigen [3]. Die Gonadotoxizität durch Strahlentherapie ist erheblich, wobei die sterilisierende Dosis bei Frauen mit zunehmendem Alter immer kleiner wird [3]. Im Bereich der Chemotherapie kann insbesondere Cyclophosphamid in höherer Dosierung den Effekt einer chemischen Kastration haben (Abb. 1).
Die Rate an Amenorrhöen nach Chemotherapie (Doxorubicin/ Cyclophosphamid (AC), AC $\rightarrow \mathrm{T}$ (Docetaxel), Cyclophosphamid, Methotrexat und 5-Fluoruracil (CMF)) beim Mammakarzinom betrug in einer prospektiven Studie [4] altersabhängig bis zu 75\% (6 Monate $\mathrm{AC} \rightarrow \mathrm{T}$ bei Frauen $\geq 40$ Jahre). Auch ein Wiedereinsetzen der Monatsblutung wurde in Abhängigkeit von der Dauer der Chemotherapie und dem Alter der Patientin immer unwahrscheinlicher. Da die ovarielle Reserve nicht regenerationsfähig ist und im Laufe der Zeit abnimmt [5], kommt es bei einem therapiebedingten Verlust auch nur eines Teils der Eizellen relativ schnell zu einer ovariellen Insuffizienz und früher Menopause. Auch bei einer Krebserkrankung im Kindesalter und einer damit verbundenen medikamentösen Therapie setzt die Menopause früher ein als bei gesunden Geschwistern [6]. Da heute bei Frauen im Alter um die 30 Jahre die Familienplanung noch nicht begonnen, geschweige denn abgeschlossen ist, wirkt sich eine auch nur partielle Schädigung der Ovarien sehr nachteilig aus. In einer populationsbasierten Untersuchung aus Schottland wurden Frauen (<39 Jahre) nach überstandener Brustkrebserkrankung seltener schwanger als altersgleiche Kontrollen [7]. Auch bei Männern kann eine Krebstherapie die Gonaden schädigen, dies gilt sowohl für Strahlentherapie (> 2,5 Gy) als auch für verschiedene Chemotherapien (Abb. 2). Insgesamt ist die Datenlage zu therapiebedingten Fertilitätsverlusten beim Mann nicht sehr umfangreich.

In Fällen, in denen die Krebserkrankung ein Abwarten erlaubt, kann durch Kryokonservierung von Eizellen, Ovarialgewebe bzw. Spermien die Fruchtbarkeit erhalten werden. Das Verfahren ist ab der Pubertät problemlos anzuwenden und kann im Prinzip auch schon im Kindesalter angeboten werden. Nach Daten von FertiPROTEKT 2015 [8] und der Gesellschaft der Epidemiologi-

\section{KARGER}

(c) 2019 S. Karger AG, Basel

Fax +497614520714 


\begin{tabular}{ll}
\hline Risiko & Regime/Substanz \\
\hline Hohes Risiko & CMF, CEF, CAF, TAC $\times 6$ bei Frauen $\geq 40$ Jahre \\
(>80\%iges Risiko für eine & Konditionierung für Stammzellentransplantation \\
permanente Amenorrhö) & (insbesondere myeloablative Konditionierung) \\
& BEACOPP $\times 6-8$ bei Frauen $>35$ Jahre \\
\hline Intermediäres Risiko & CMF, CEF, CAF, TAC $\times 6$ bei Frauen $30-39$ Jahre \\
(40-60\%iges Risiko für eine & AC $\times 4$ bei Frauen $\geq 40$ Jahre \\
permanente Amenorrhö) & AC oder EC $\times 4 \rightarrow$ Taxan \\
& BEACOPP $\times 6-8$ bei Frauen 25-35 Jahre \\
& CHOP $\times 6$ bei Frauen $\geq 35$ Jahre \\
& Knochen- und Weichteilsarkom-typische Therapie \\
\hline Niedriges Risiko & CMF, CEF, CAF, TAC $\times 6$ bei Frauen $\leq 30$ Jahre \\
(<20\%iges Risiko für eine & AC $\times 4$ bei Frauen $\leq 40$ Jahre \\
permanente Amenorrhö) & BEACOPP $\times 6-8$ bei Frauen $<25$ Jahre \\
& ABVD $\times 2-4$ \\
& CHOP $\times 6$ bei Frauen $<35$ Jahre \\
& CVP \\
& AML-typische Therapie (Anthrazyklin/Cytarabin) \\
& ALL-typische Therapie (multi-agent) \\
& FOLFOX bei Frauen $\leq 40$ Jahre \\
\hline Sehr niedriges oder kein & Methotrexat \\
Risiko für eine permanente & Fluorouracil \\
Amenorrhö & Vincristin \\
\hline
\end{tabular}

\begin{tabular}{|c|c|c|c|}
\hline Effekt & Therapie & $\begin{array}{l}\text { Kumulative } \\
\text { Dosis }\end{array}$ & $\begin{array}{l}\text { Wirkmechanis- } \\
\text { mus }\end{array}$ \\
\hline \multirow{6}{*}{$\begin{array}{l}\text { Prolongierte } \\
\text { Azoospermie }\end{array}$} & Radiotherapie & 2,5 Gy & DNA-Brüche \\
\hline & Cyclophosphamid & $19 \mathrm{~g} / \mathrm{m}^{2}$ & Alkylanz \\
\hline & Chlorambucil & $1,4 \mathrm{~g} / \mathrm{m}^{2}$ & Alkylanz \\
\hline & Cisplatin & $500 \mathrm{mg} / \mathrm{m}^{2}$ & Alkylanz \\
\hline & Melphalan & $140 \mathrm{mg} / \mathrm{m}^{2}$ & Alkylanz \\
\hline & Procarbazin & $4 \mathrm{~g} / \mathrm{m}^{2}$ & Alkylanz \\
\hline \multirow{2}{*}{$\begin{array}{l}\text { Azoospermie nach Krebser- } \\
\text { krankung im Kindesalter }\end{array}$} & BCNU & $1 \mathrm{~g} / \mathrm{m}^{2}$ & Alkylanz \\
\hline & CCNU & $500 \mathrm{mg} / \mathrm{m}^{2}$ & Alkylanz \\
\hline \multirow{4}{*}{$\begin{array}{l}\text { Als Kombinationstherapeutika } \\
\text { eingesetzte Präparate, die zur } \\
\text { prolongierten Azoospermie führen }\end{array}$} & Busulfan & $600 \mathrm{mg} / \mathrm{kg}$ & Alkylanz \\
\hline & Ifosfamid & $42 \mathrm{~g} / \mathrm{m}^{2}$ & Alkylanz \\
\hline & Actinomycin D & & $\begin{array}{l}\text { DNA-Strang- } \\
\text { brüche }\end{array}$ \\
\hline & Mechlorethamin & & Alkylanz \\
\hline \multirow{4}{*}{$\begin{array}{l}\text { Nur in Kombination mit obigen } \\
\text { Präparaten dauerhafte Azoo- } \\
\text { spermie, alleinige Gabe nur pas- } \\
\text { sagere Azoospermie }\end{array}$} & Adriamycin & $770 \mathrm{mg} / \mathrm{m}^{2}$ & $\begin{array}{l}\text { DNA-Strang- } \\
\text { brüche }\end{array}$ \\
\hline & Thiotepa & $400 \mathrm{mg} / \mathrm{m}^{2}$ & Alkylanz \\
\hline & Cytosin-Arabinosid & $1 \mathrm{~g} / \mathrm{m}^{2}$ & $\begin{array}{l}\text { Nukleosid- } \\
\text { Analogon }\end{array}$ \\
\hline & Vinblastin & $50 \mathrm{mg} / \mathrm{m}^{2}$ & $\begin{array}{l}\text { Mikrotubulin- } \\
\text { Inhibitor }\end{array}$ \\
\hline
\end{tabular}

Abb. 1. Gonodotoxizität durch Tumortherapien bei Frauen; modifiziert nach [3].
Abb. 2. Gonadotoxizität durch Tumortherapien bei Männern; modifiziert nach [3]. schen Krebsregister in Deutschland (GEKID) [9] entscheiden sich nur 8,4\% der jüngeren Frauen mit Krebserkrankung für Maßnahmen zum Erhalt der Fruchtbarkeit. Ein Grund ist unter anderem, dass bis zum Inkrafttreten des Terminservice- und Versorgungsgesetz (TSVG) im Mai 2019 die Kosten der Fruchtbarkeitserhaltung von den gesetzlichen Krankenkassen nicht übernommen wurden. Die Kosten belaufen sich auf bis zu 4300 Euro (Kryokonservierung befruchteter Eizellen). Hinzu kommen die Kosten für die Lagerung in Höhe von etwa 300 Euro pro Jahr.

Die DGHO und die Deutsche Stiftung für junge Erwachsene mit Krebs hatten das Thema über Jahre hinweg beharrlich auf die gesundheitspolitische, mediale und öffentliche Agenda gebracht 
Abb. 3. Fatigue in einer AYA-Kohorte; Anteil der Langzeitüberlebenden mit überschwelligen Fatigue-Symptomen für $\geq 6$ Monate; modifiziert nach [12]. ${ }^{*}$ Unterschied signifikant gegenüber der Melanom-Gruppe.

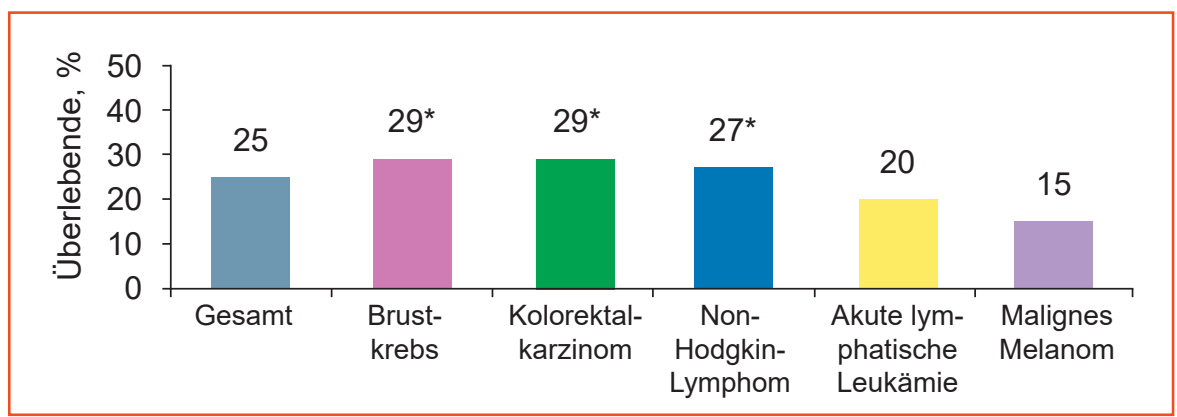

und damit zur Gesetzesänderung beigetragen. Durch das TSVG wurde eine Änderung im $\$ 27$ des SGB V beschlossen. Dort heißt es nun: «(Versicherte haben) Anspruch auf Kryokonservierung von Ei- oder Samenzellen oder von Keimzellgewebe sowie auf die dazugehörigen medizinischen Maßnahmen, wenn die Kryokonservierung wegen einer Erkrankung und deren Behandlung mit einer keimzellschädigenden Therapie medizinisch notwendig erscheint, um spätere medizinische Maßnahmen zur Herbeiführung einer Schwangerschaft nach Absatz 1 vornehmen zu können». Und weiter: «Bei Vorliegen der leistungsrechtlichen Voraussetzungen übernimmt die gesetzliche Krankenversicherung danach künftig die Kosten für die erforderlichen Leistungen im Zusammenhang mit der Kryokonservierung, insbesondere Entnahme, Aufbereitung, Lagerung und ein späteres Auftauen, in vollem Umfang im Rahmen des Sachleistungsprinzips. Dabei ist der Anspruch nach Absatz 4 nicht an die für die spätere künstliche Befruchtung geltenden Voraussetzungen der unteren Altersgrenze von 25 Jahren und der Ehe geknüpft.» Aktuell wird beim Gemeinsamen Bundessausschuss (G-BA) eine entsprechende Richtlinie erarbeitet.

\section{Problemfeld Fatigue}

\section{Referent: Markus Horneber, Nürnberg}

Der Begriff Fatigue beschreibt ein unangenehmes, andauerndes Gefühl der Erschöpfung, Müdigkeit und mangelnder Energiereserven. Die Beschwerden stehen in keinem Verhältnis zum Ausmaß vorangehender Belastungen und bilden sich durch Ruhe und Schlaf nicht in gewohntem Maß zurück. Sie sind mit einer verminderten körperlichen und mentalen Leistungsfähigkeit verbunden, beeinflussen Stimmung, Antrieb und Verhalten und beeinträchtigen das Alltagsleben der Betroffenen [10].

Zur Häufigkeit und Ausprägung von Fatigue sowie deren Auswirkungen auf das Leben junger Erwachsener mit Krebserkrankungen (AYA, adolescents and young adults with cancer) ist bisher wenig bekannt und die Autoren einer Übersichtsarbeit aus dem Jahr 2017 stellen erheblichen Forschungsbedarf fest [11]. Eine aktuelle Querschnittserhebung hat bei 1088 Langzeitüberlebenden nach einer Krebserkrankung im Alter von 19-39 Jahren Daten zur Fatigue erhoben [12]. Die Studienteilnehmer waren zu 74\% weiblich und zum Zeitpunkt der Erhebung im Durchschnitt
49 Jahre alt. Die Krebsdiagnose lag im Mittel 15 Jahre zurück. Eine Fatigue lag vor, wenn die Angaben auf der Chalder Fatigue Scale (7 Fragen $\mathrm{zu}$ "physical fatigue», 4 Fragen $\mathrm{zu}$ «mental fatigue», Wertebereich $0-11$ ) für den vorangehenden Monat einen Wert von $\geq 4$ ergaben. Dies war insgesamt bei etwa einem Viertel der Teilnehmer der Fall (Abb. 3); etwas häufiger von Fatigue betroffen waren Patienten nach Brustkrebs, Darmkrebs oder NonHodgkin-Lymphomen. Das Risiko für das Auftreten von Fatigue war umso höher, je mehr Modalitäten zur onkologischen Therapie eingesetzt worden waren. Bei $60 \%$ der von Fatigue Betroffenen bestanden die Beschwerden seit der onkologischen Therapie, mithin seit vielen Jahren. Knapp die Hälfte der Betroffenen (45\%) gab an, dass sich die Beschwerden im Verlauf der Zeit verschlechtert hätten, ein Drittel (35\%) empfand eine Besserung und 20\% sahen keine Änderung über die Zeit.

Auch in einer Studie mit jungen Lymphom-Patienten war die Lebensqualität noch viele Jahre nach überstandener Erkrankung signifikant gegenüber Gleichaltrigen vermindert. Fatigue und kognitive Einschränkung wurden dabei als besonders belastend beschrieben und hatten auch nachteilige Auswirkungen auf den Beruf und das Sozialleben [13].

\section{Regelmäßiges Fatiguescreening}

Fatiguesymptome können sich in individueller Form körperlich, affektiv oder mental ausprägen, zu jedem Zeitpunkt der Erkrankung auftreten, zeitlich begrenzt sein oder persistieren. Nach aktuellen Leitlinienempfehlungen sollten Krebspatienten deshalb aktiv und regelmäßig mithilfe validierter Messinstrumente nach Fatiguesymptomen gefragt werden [10, 14]. Die Leitlinien empfehlen hierfür visuelle oder numerische Analyseskalen (VAS/ NAS), die schnell anzuwenden sind. Das Screening auf Fatigue kann auch mit weiteren Symptomen verbunden werden, insbesondere während der Behandlungsphase. In der Praxis hat sich hierfür das ESASr (Edmonton Symptom Assessment System, revidierte Fassung) als Fragebogen bewährt. Auch mehrdimensionale Instrumente können verwendet werden und liefern je nach Instrument zusätzliche Informationen (z.B. BFI (Brief Fatigue Inventory), QLQ-FA12 (EORTC Quality of Life Module Measuring Cancer-Related Fatigue)). Nachteile der mehrdimensionalen Fragebögen sind jedoch der Zeitaufwand für die Datenerfassung und deren Auswertung. 
Mit der möglichst frühen Erfassung und Therapie von Fatiguesymptomen soll einer Chronifizierung vorgebeugt werden, da das Erleben von Fatiguebeschwerden den wichtigsten Risikofaktor dafür darstellt, dass Fatigue erneut auftritt oder persistiert [15].

Eine frühzeitige Intervention bei Fatiguebeschwerden kann außerdem die Abwärtsspirale aus Fatigue, dem daraus resultierenden Verlust von Funktionalität und Belastungsfähigkeit und folgender körperlicher Dekonditionierung aufhalten. Häufig suchen Betroffene jedoch erst Hilfe, wenn die Beeinträchtigungen für sie nicht mehr anderweitig erklärbar oder die Kompensationsmöglichkeiten erschöpft sind.

\section{Behandelbare Einflussfaktoren erfassen}

Nach den Empfehlungen von Leitlinien soll bei Patienten mit Fatigue von ärztlicher Seite zunächst versucht werden, behandelbare Ursachen und Einflussfaktoren zu identifizieren. Dies erfolgt durch gezielte Anamnese und Untersuchungen, sowie einer Beurteilung der Krebserkrankung, von Komorbiditäten sowie von Symptomen, Belastungen und Funktionsstörungen [10, 14].

In Ergänzung dazu schlagen die Leitlinien eine kriterienbasierte Diagnosestellung von Cella et al. [16] vor [10] (Abb. 4). Zur Diagnose eines Fatiguesyndroms müssen danach das Kriterium A1 sowie weitere A-Kriterien und die Kriterien B-D vorliegen.

\section{Evidenzbasierte Behandlung}

Die Grundlage der Behandlung von Fatigue bildet die Information und Beratung der Betroffenen. Eine gute Hilfe sind dabei die Informationen von AYApedia auf der Onkopedia-Website [17], die Wissen über Entstehung und Einflussmöglichkeiten bei Fatigue einschließlich präventiver Strategien vermitteln und die Einbeziehung der Angehörigen anregen.
Nach den Empfehlungen von Leitlinien stehen psychosoziale und bewegungstherapeutische Interventionen im Zentrum der Behandlung. Sie haben mittlere geschätzte Effektstärken (Bewegungstherapien 0,30; psychosoziale Interventionen 0,27), können miteinander kombiniert werden und sollten sich an der individuellen Ausprägung der Beschwerden und dem Ausmaß der funktionellen Beeinträchtigung orientieren. Psychosoziale Interventionen dienen dabei der Förderung des Selbstmanagements, dem Umgang mit Stressoren, der Stärkung von Ressourcen, der Verbesserung der Körperwahrnehmung, der Aufmerksamkeitssteuerung sowie Änderungen von dysfunktionalen Kognitionen und maladaptiven Verhaltensweisen. Als evaluiertes Schulungsmanual und Selbstmanagementprogramm steht hier z.B. «Fatigue individuell bewältigen» (FIPS) zur Verfügung [18].

Bewegungstherapeutische Interventionen sind als körperliches Training (Kraft- und Ausdauertraining) oder achtsamkeitsbasierte Bewegung (Yoga, Qigong, Taiji) wirksam. Beide Interventionsformen erzielen positive Effekte schon nach kurzen Anwendungs- bzw. Übungszeiten [19, 20]. Für medikamentöse Behandlungen sprechen Leitlinien derzeit keine Empfehlungen aus, sehen aber Einsatzmöglichkeiten in einzelnen Fällen [10].

\section{Ausblick}

Zur Umsetzung der in den Leitlinien ausgesprochenen Empfehlungen zur Prävention, Diagnostik und Therapie von Fatigue bei Krebserkrankungen ist der Aufbau von Versorgungs- und Forschungsstrukturen an den Behandlungszentren erforderlich. Hiermit würde auch der Prävalenz des Beschwerde- und Krankheitsbildes und seinen individuellen und gesellschaftlichen Auswirkungen entsprochen. Die Gründung des «Charité Fatigue Centrum» im März 2019 ist ein wichtiger Schritt in diese Richtung.

\begin{tabular}{|l|l|}
\hline A1 und mindestens fünf der folgenden Symptome \\
\hline A1 & $\begin{array}{l}\text { Deutliche Müdigkeit, Energieverlust oder verstärktes Ruhebedürfnis inadäquat zur } \\
\text { Aktivität }\end{array}$ \\
\hline A2 & Beschwerden allgemeiner Schwäche oder schwerer Glieder \\
\hline A3 & Verminderte Fähigkeit zu Konzentration und Aufmerksamkeit \\
\hline A4 & Verringerte(s) Motivation oder Interesse an Alltagsaktivitäten \\
\hline A5 & Schlaflosigkeit oder vermehrter Schlaf \\
\hline A6 & Schlaf wird nicht als erholsam und regenerierend erlebt \\
\hline A7 & Notwendigkeit starker Anstrengung, um Inaktivität zu überwinden \\
\hline A8 & $\begin{array}{l}\text { Ausgeprägte emotionale Reaktionen auf Symptomatik } \\
\text { (z.B. Frustration oder Reizbarkeit) }\end{array}$ \\
\hline A9 & Durch Müdigkeit bedingte Schwierigkeiten, alltägliche Aufgaben zu erledigen \\
\hline A10 & Probleme mit dem Kurzzeitgedächtnis \\
\hline A11 & Mehrere Stunden anhaltendes Unwohlsein nach Anstrengung \\
\hline B: Erheblicher Leidensdruck oder Einschränkung in sozialen Bereichen \\
\hline C: Eindeutiger Bezug zur Tumorerkrankung oder -behandlung \\
\hline D: Die Symptomatik ist nicht primäre Folge psychischer Komorbiditäten \\
\hline
\end{tabular}

Abb. 4. Diagnose der Fatigue; modifiziert nach [16]. 


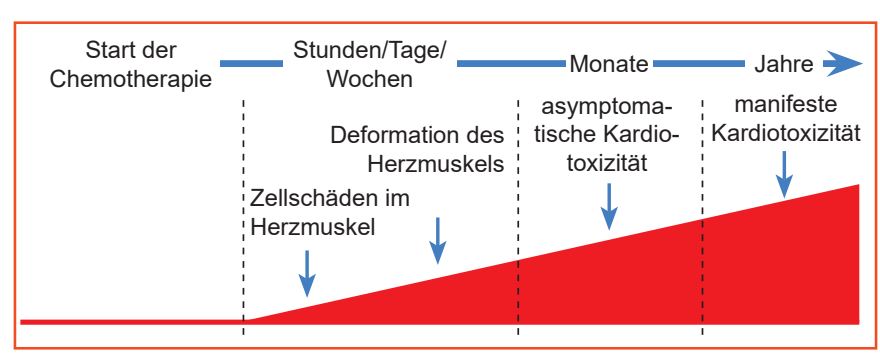

Abb. 5. Entwicklung der Kardiotoxizität nach Chemotherapie; modifiziert nach [23].

Im März 2018 wurde in der DGHO ein Arbeitskreis «Fatigue» gegründet, der es sich - auch mit Blick auf die Unterstützung der AYA - zum Ziel gesetzt hat, den interdisziplinären Dialog zu fördern, gezielte Fortbildungsangebote zu entwickeln, Forschungsprojekte zu initiieren und durchzuführen, eine Leitlinie zu erstellen und eng mit nationalen und internationalen Fachgesellschaften zum Thema Fatigue zusammenzuarbeiten.

\section{Problemfeld Kardiotoxizität}

\section{Referent: Roman Pfister, Köln}

Die Zahl der Menschen, die mit überstandener oder chronischer Krebserkrankung leben, ist in den vergangenen 20 Jahren deutlich gestiegen [21]. Dennoch ist ihre Lebenserwartung im Vergleich zur Allgemeinbevölkerung reduziert [22]. Die vorzeitigen Todesfälle sind hauptsächlich auf die primäre Tumorerkrankung oder ein Zweitmalignom zurückzuführen, während 7\% der Patienten an kardiovaskulären Ursachen verstarben. Da bereits in der Frühphase der Therapie die Grundlage für spätere kardiovaskuläre Komplikationen gelegt wird [23], ist ein regelmäßiges kardiales Monitoring ab Beginn der Therapie sinnvoll (Abb. 5). Dies beschränkt sich in der Routine häufig auf die Bestimmung der Ejektionsfraktion. Ein Abfall um mehr als 10\% auf unter 50\% (unterer Normwert) ist ein Signal für therapeutisches Handeln. In der St. Judes Lifetime-Kohortenstudie [24] konnte jedoch gezeigt werden, dass es außer diesen Veränderungen noch weitere Funktions- störungen gibt, die auf eine Schädigung durch eine Chemotherapie hinweisen können (Abb. 6). Für die Studie wurden die Langzeitverläufe von fast 2000 Patienten ausgewertet, die mit einer anthrazyklinhaltigen Chemotherapie und/oder Thoraxbestrahlung behandelt worden waren. Die kardiovaskulären Auffälligkeiten markieren ein erhöhtes Herzinsuffizienzrisiko, wie Studien bei Nicht-Krebspatienten gezeigt haben. Ein pragmatischer Ansatz wäre, bei anthrazyklinhaltigen Therapien grundsätzlich eine Prävention mit einem ACE-Hemmer ( $\mathrm{ACE}=$ Angiotensin konvertierendes Enzym) zu beginnen, was allerdings auch mit Nebenwirkungen verbunden sein kann. Alternativ wurde eine "selektive», d.h. nur bei erhöhten Troponin-Werten indizierte Primärprävention im Rahmen der ICOSone-Studie bei Patienten mit Anthrazyklintherapie geprüft [25]. Die Ergebnisse legen nahe, dass eine nur für Hochrisikopatienten konzipierte Prävention mit einem ACEHemmer (in diesem Fall Enalapril) ausreicht.

Ein weiterer Ansatz besteht darin, Patienten mithilfe der Bildgebung für eine kardiovaskuläre Prävention zu selektieren (noch laufende SUCCOUR-Studie).

\section{Kardiotoxizität manifestiert sich erst spät}

Die häufigste kardiale Todesursache nach Krebs ist die koronare Herzerkrankung (KHK), wie eine Auswertung von 34000 Patienten mit pädiatrischen Tumoren und Langzeitüberleben von mindestens 5 Jahren gezeigt hat [26]. Das erhöhte Mortalitätsrisiko von ehemaligen Krebspatienten zeigt sich erst im höheren Lebensalter, wie den Daten zum Langzeit-Follow-up zu entnehmen ist. Möglicherweise ist das auf die kardiale Vorschädigung in Verbindung mit einer verringerten Toleranz gegenüber altersbedingten Stressoren zurückzuführen. Dass die kardiale Morbidität nach Krebs erhöht ist, wurde auch in der Childhood Cancer Survivors Study nachgewiesen [27]. Darin wurden bei ehemaligen Krebspatienten 4-8-mal häufiger kardiale Erkrankungen diagnostiziert als bei ihren nicht an Krebs erkrankten Geschwistern. Prädiktiv für die erhöhte kardiale Morbidität waren auch hier anthrazyklinhaltige Therapien und eine Radiatio. Beide waren insbesondere mit einem erhöhten Risiko für Herzinsuffizienz assoziiert.

Abb. 6. Präklinische Veränderungen nach Krebs;

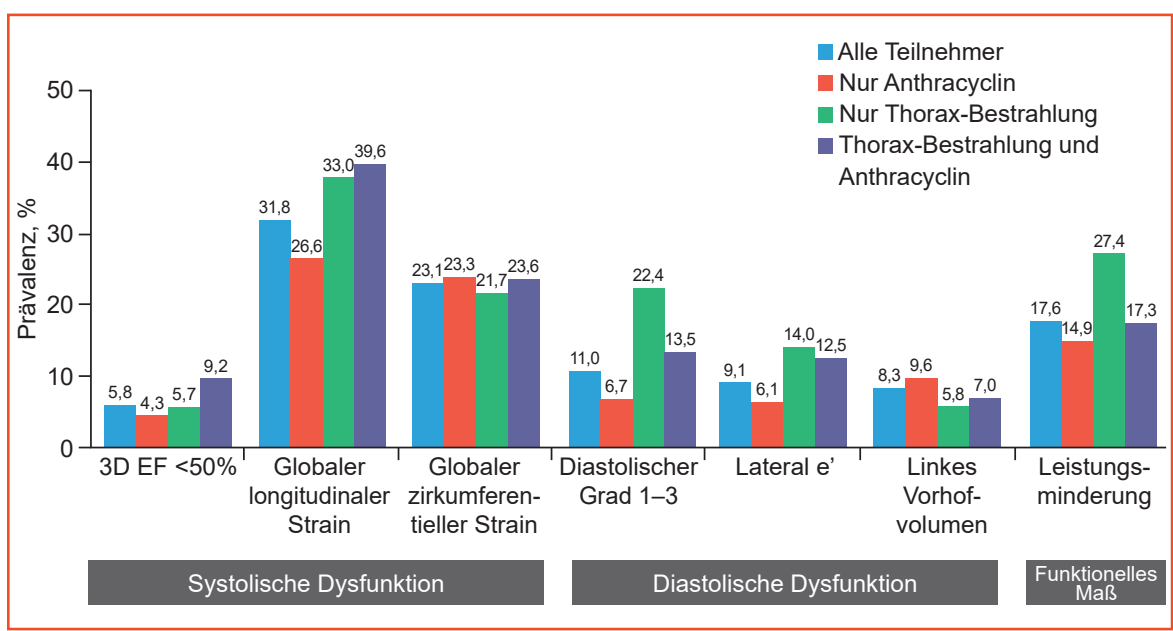
modifiziert nach [25]. EF = Ejektionsfraktion 
Die Kardiotoxizität als potenzielle Therapiekomplikation tritt heute nicht mehr so häufig auf wie in den 1970er- und 1980erJahren [26]. Dazu mögen neben modernen Dosis- und Bestrahlungsregimen auch verbesserte Nachsorgeprogramme beigetragen haben. Aktuell weist eine Datenanalyse von fast 350000 Patientinnen mit chemo- bzw. strahlentherapeutisch behandeltem Brustkrebs und 8-jähriger Nachbeachtung einen nochmaligen Rückgang der kardialen Mortalität für den Zeitraum 2006-2010 im Vergleich zu den Jahren 2000-2005 nach [28].

Inwieweit eine «modernere» Strahlentherapie das Risiko für Kardiotoxizität gesenkt hat, ist bislang wenig untersucht. Eine Studie dokumentiert 190 Fälle von neu aufgetretener Herzinsuffizienz bei Frauen mit Mammakarzinom und Radiatio (in den Jahren 1998-2013) [29]. Die Patientinnen waren bei der Bestrahlung im Mittel 70 Jahre alt und hatten nach durchschnittlich 6 Jahren eine Herzinsuffizienz (überwiegend mit erhaltender Ejektionsfraktion) entwickelt. Das Risiko für Herzinsuffizienz war abhängig von der applizierten Strahlendosis, dies galt auch nach Adjustierung auf Alter, Tumorstadium und andere Risikofaktoren.

\section{Genetische Disposition spielt eine Rolle}

$\mathrm{Ob}$ und wie stark sich eine Kardiotoxizität herausbildet, hängt auch von der individuellen genetischen Disposition ab. Dies konnte an patientenspezifischen Kardiomyozyten, die aus humanen induzierbaren pluripotenten Stammzellen kultiviert wurden, nachgewiesen werden [30]. Darüber hinaus gibt es gemeinsame Risikofaktoren für Gefäßerkrankungen und Krebs (etwa das Rauchen), was ebenfalls eine Kardiotoxizität begünstigen kann [31]. Die Überschneidung des Risikoprofils macht es aber auch möglich, durch Beeinflussung der modifizierbaren Risikofaktoren die Therapie herzverträglicher zu machen. Dies gilt besonders für das metabolische Syndrom und die nichtkontrollierte arterielle Hypertonie, von denen laut dem Cardiac Oncology Toxicity (COT) Registry der European Society of Cardiology (ESC) etwa 19\% bzw. 15\% der älteren Krebspatienten bei Registereinschluss betroffen waren. In einer deutschen Studie mit 1000 Teilnehmern mit einer mindestens 15 Jahre zurückliegenden Krebserkrankung im Kindesalter wurde eine erhöhte Inzidenz von Fettstoffwechselstörungen und Hypertonie im Vergleich zur Allgemeinbevölkerung festgestellt [32]. Die Krankheiten traten im Schnitt 8 bzw. 6 Jahre früher auf als in altersgleichen Kohorten, weshalb hier von einem «akzelerierten kardiovaskulären Altern» bei Krebs gesprochen werden kann. Besonders bedenklich ist, dass Patienten, die nach anthrazyklinhaltiger Chemotherapie oder Bestrahlung eine Hypertonie, Dyslipidämie, Diabetes oder Adipositas entwickeln, ein stark erhöhtes Risiko für schwere Herzerkrankungen wie KHK, Herzinsuffizienz und Klappenerkrankungen haben [33]. Um die langfristig ungünstige kardiovaskuläre Prognose zu verbessern, geht es neben der Therapieoptimierung auch um eine verbesserte Früherkennung und Prävention kardiovaskulärer Komplikationen. Am einfachsten ist dies umzusetzen, indem bei kardiovaskulär gefährdeten Krebspatienten eine lebenslange Nachsorge etabliert wird.

\section{Disclosure Statement}

D. Lüftner, M. Horneber und R. Pfister erklären, dass keine Interessenkonflikte vorliegen.

\section{References}

1 Leuteritz K et al.: Life satisfaction in young adults with cancer and the role of sociodemographic, medical, and psychosocial factors: results of a longitudinal study. Cancer 2018;124:4374-4382

2 DGHO: Gesundheitspolitische Schriftenreihen, Band 11, Fertilitätserhalt, November 2017.

3 S2k-Leitlinie «Fertilitätserhaltung bei onkologischen Therapien", AWMF-Registernummer 015-082, Stand 01.11.2017.

4 Sukumvanich $\mathrm{P}$ et al.: Incidence and time course of bleeding after long-term amenorrhea after breast cancer treatment: a prospective study. Cancer 2010;116: 3102-3111.

5 Wallace WH, Kelsey TW: Human ovarian reserve from conception to the menopause. PLoS One 2010; 5:e8772.
6 Sklar CA et al.: Premature menopause in survivors of childhood cancer: a report from the childhood cancer survivor study. J Natl Cancer Inst 2006;98:890-896.

7 Anderson RA et al.: The impact of cancer on subsequent chance of pregnancy: a population-based analysis. Hum Reprod 2018;33:1281-1290.

8 https://static1.squarespace.com/static/ $560 a 328 f e 4 b 0 e 8 c 4 f 373857 e / t / 57206 c 853 c 44 d 81 e a 19 e 7$ 90b/1461742728020/registerdaten_fertiprotekt_2015. pdf.

9 https://atlas.gekid.de/CurrentVersion/atlas.html.

10 National Comprehensive Cancer Network (NCCN): Cancer-related fatigue. Clinical Practice Guidelines in Oncology (NCCN Guidelines). www.nccn.org, 2019.

11 Nowe E et al.: Cancer-related fatigue in adolescents and young adults: a systematic review of the literature. Crit Rev Oncol Hematol 2017;118:63-69.
12 Bøhn SH et al.: Chronic fatigue and associated factors among long-term survivors of cancers in young adulthood. Acta Oncol 2019;30:1-10.

13 Husson $\mathrm{O}$ et al.: Adolescent and young adult (AYA) lymphoma survivors report lower health-related quality of life compared to a normative population: results from the PROFILES registry. Acta Oncol 2017;56: 288-294.

14 S3-Leitlinie Diagnostik, Therapie und Nachsorge des Hodgkin Lymphoms bei erwachsenen Patienten, Version 2.0, Juni 2018. AWMF-Registernummer: 018/029.

15 Kreissl S et al.: Cancer-related fatigue in patients with and survivors of Hodgkin's lymphoma: a longitudinal study of the German Hodgkin Study Group. Lancet Oncol 2016;17:1453-1462.

16 Cella D et al.: Progress toward guidelines for the management of fatigue. Oncology (Williston Park) 1998; $12: 369-377$ 
17 https://www.onkopedia.com/de/ayapedia/guidelines/ fatigue/@@view/html/index.html.

18 Reif $\mathrm{K}$ et al.: A patient education program is effective in reducing cancer-related fatigue: a multi-centre randomised two-group waiting-list controlled intervention trial. Eur J Oncol Nurs 2013;17:204-213.

19 Carayol M et al.: Population-, intervention- and methodology-related characteristics of clinical trials impact exercise efficacy during adjuvant therapy for breast cancer: a meta-regression analysis. Psychooncology 2015;24:737-747.

20 Mustian KM et al.: Comparison of pharmaceutical, psychological, and exercise treatments for cancer-related fatigue: a meta-analysis. JAMA Oncol 2017;3: 961-968.

21 Shapiro CL: Cancer survivorship. N Engl J Med 2018; 379:2438-2450.

22 Armstrong GT et al.: Late mortality among 5-year survivors of childhood cancer: a summary from the Childhood Cancer Survivor Study. J Clin Oncol 2009;27: 2328-2338.
23 Cardinale D, Biasillo G, Cipolla CM: Curing cancer, saving the heart: a challenge that cardioncology should not miss. Curr Cardiol Rep 2016;18:51.

24 Armstrong GT et al.: Comprehensive echocardiographic detection of treatment-related cardiac dysfunction in adult survivors of childhood cancer: results from the St. Jude Lifetime Cohort Study. J Am Coll Cardiol 2015;65:2511-2522.

25 Cardinale D et al.: Anthracycline-induced cardiotoxicity: a multicenter randomised trial comparing two strategies for guiding prevention with enalapril: the International CardioOncology Society-one trial. Eur J Cancer 2018;94:126-137.

26 Fidler MM et al.: Population-based long-term cardiacspecific mortality among 34489 five-year survivors of childhood cancer in Great Britain. Circulation 2017; 135:951-963.

27 Mulrooney DA et al.: Cardiac outcomes in a cohort of adult survivors of childhood and adolescent cancer: retrospective analysis of the Childhood Cancer Survivor Study cohort. BMJ 2009;339:b4606.
28 Weberpals J et al.: Long-term heart-specific mortality among 347476 breast cancer patients treated with radiotherapy or chemotherapy: a registry-based cohort study. Eur Heart J 2018;39:3896-3903.

29 Saiki $\mathrm{H}$ et al.: Risk of heart failure with preserved ejection fraction in older women after contemporary radiotherapy for breast cancer. Circulation 2017; 135:1388 1396

30 Burridge PW et al.: Human induced pluripotent stem cell-derived cardiomyocytes recapitulate the predilection of breast cancer patients to doxorubicin-induced cardiotoxicity. Nat Med 2016;22:547-556.

31 Koene R et al.: Shared risk factors in cardiovascular disease and cancer. Circulation 2016;133:1104-1114.

32 Faber J et al.: Burden of cardiovascular risk factors and cardiovascular disease in childhood cancer survivors: data from the German CVSS-study. Eur Heart J 2018; 39:1555-1562.

33 Armstrong GT et al.: Modifiable risk factors and major cardiac events among adult survivors of childhood cancer. J Clin Oncol 2013;31:3673-3680. 